\title{
A Model Predictive Control Scheme with Ultimate Bound for Economic Optimization
}

\author{
Andrea Alessandretti ${ }^{1}{ }^{2}$, A. Pedro Aguiar ${ }^{2}{ }^{3}$ and Colin N. Jones ${ }^{1}$
}

\begin{abstract}
This paper presents a Model Predictive Control (MPC) scheme for nonlinear continuous-time systems where an economic stage cost, which is not a measure of the distance to a desired set point, is combined with a classic stabilizing stage cost. The associated control strategy leads to a closedloop behavior that compromises, in a seamless way, between the convergence of the closed-loop state trajectory to a given steadystate and the minimization of the economic cost. More precisely, we derive a set of sufficient conditions under which the closedloop state trajectory is ultimately bounded around the desired steady-state, with the size of the bound being proportional to the strength of the economic cost. Numerical results show the effectiveness of the proposed scheme on a target estimation and tracking control problem.
\end{abstract}

\section{INTRODUCTION}

In a Tracking MPC scheme, where the main goal is to drive the state vector to a desired steady-state or state trajectory, the performance index is chosen to penalize the distance from the current state to the desired one. An overview of methods that utilize the so-called terminal set and terminal cost can be found in [22], [26]. We refer to [16] for the terminal-set-free case, where the terminal set is not considered and stability is guaranteed by properly choosing a long horizon. Insightful properties of finite horizon optimal controls are presented in [19]. For the continuous-time quasiinfinite horizon approach we refer to [9] and [14].

In Economic MPC, the main objective is the minimization of a given economic performance index. Since the value function used in Tracking MPC has proved to be inadequate for the economic case, in [11], [7], [8], [5], a dissipativity property of the system is exploited to define a rotated value function that, in contrast to the first, manifests the desired monotonic decrease as the state approaches the economically optimal steady-state of the system. This function is then utilized to derive sufficient conditions for convergence to an economically optimal steady-state using terminal equality, [11], [7], and inequality, [8], [5], constraints. The terminal constraints are not considered in [15] where sufficient conditions on the horizon length are provided to guarantee convergence to an arbitrarily small neighborhood of the optimal steady-state. Convergence properties to the economically optimal steady-state for variations of the performance index are addressed in [13].

Other approaches that avoid the use of the dissipativity property of the system have been presented in the literature.

\footnotetext{
${ }^{1}$ École Polytechnique Fédérale de Lausanne (EPFL), Lausanne, Switzerland.

${ }^{2}$ Institute for Systems and Robotics (ISR), Instituto Superior Tecnico (IST), Lisbon, Portugal.

${ }^{3}$ Faculty of Engineering, University of Porto (FEUP), Porto, Portugal.
}

In [12], [23], the authors, in order to guarantee closed-loop convergence to a steady-state, employ a generalized terminal set, consisting of all reachable steady-states, and enforce the terminal cost to not increase from one solution of the MPC optimization problem to the other. In [17] a given CLF defined over the whole desired region of attraction of the MPC controller is exploited to initially constrain the closedloop trajectory within a bounded region, and then, at an arbitrary given time, to enforce convergence to the desired steady-state. In [21], essentially, the robustness margin of a predefined steady-state it traded for the increase of economic performance while still conserving closed loop stability.

On the same line, avoiding the use of the dissipativity property of the system, the combination of a stabilizing stage cost, from Tracking MPC, with an economic stage cost is used in this paper to derive a set of sufficient conditions for ultimate boundedness of the closed-loop state trajectories around a desired steady-state. As main advantage, as shown in a numerical example, by only enforcing ultimate boundedness, the proposed scheme allows, for instance, periodic behaviors that naturally arise in many economic problems. This approach is suitable for a variety practical applications where the compromise between economic optimization and convergence to a predefined steady-state corresponds with the desired behaviour (see the motivating works [24], [10], [1], [25]). Moreover, by introducing the stabilizing stage cost, neither the assumption of the dissipativity of the system, e.g, [13], [11], [7], [8], [15], [5], nor constraints on the decrease of the terminal cost, [12], [23], are required. This work is on the same line of [21], [3] where stability and convergence, respectively, are further relaxed to ultimate boundedness for the sake of economic optimization.

The organization of the paper is as follows. In Section II the MPC law and the control problem are introduced. The convergence result is presented in Section III, followed by Section IV with an application of the proposed scheme on a target estimation and tracking control problem.

\section{PROBLEM DEFINITION}

Consider the dynamical system

$$
\dot{x}(t)=f(x(t), u(t)), \quad x(0)=x_{0}, \quad t \geq 0
$$

where $x(t) \in \mathbb{R}^{n}$ and $u(t) \in \mathbb{R}^{m}$ are the state and the input vectors at time $t$, respectively, and $x_{0}$ is the initial state vector. The state and input are constrained as

$$
x(t) \in \mathcal{X} \subseteq \mathbb{R}^{n}, \quad u(t) \in \mathcal{U} \subseteq \mathbb{R}^{m}
$$


where we denote by $\mathcal{X}$ and $\mathcal{U}$ the state and input constraint sets, respectively. Next, the MPC optimization problem $\mathcal{P}(z)$ is defined, with $z \in \mathcal{X}$ and where for a generic trajectory $x(\cdot)$, the term $x\left(\left[t_{1}, t_{2}\right]\right)$ denotes the trajectory considered in the time interval $\left[t_{1}, t_{2}\right]$. Moreover, the notation $x(\cdot ; z)$ is used whenever we want to make explicit the dependence of the trajectory $x(\cdot)$ on the optimization problem parameter $z$. For the sake of simplicity, the dependence on time and parameter is dropped whenever it is clear from the context.

Definition 1 (Open-loop MPC optimization problem):

Given a vector $z \in \mathbb{R}^{n}$ and an horizon length $T \in \mathbb{R}_{>0}$, the open-loop MPC optimization problem $\mathcal{P}(z)$ consists in finding the optimal control trajectory $\bar{u}^{*}([0, T])$ that solves

$$
\begin{array}{rlr}
J_{T}^{*}(z)= & \min _{\bar{u}([0, T])} J_{T}(z, \bar{u}([0, T])) & \\
\text { s.t. } & \dot{\bar{x}}(\tau)=f(\bar{x}(\tau), \bar{u}(\tau)) & \\
& \bar{x}(0)=z, \bar{x}(T) \in \mathcal{X}_{a u x} & \\
& (\bar{x}(\tau), \bar{u}(\tau)) \in \mathcal{X} \times \mathcal{U} & \forall \tau \in[0, T]
\end{array}
$$

with

$$
J_{T}(z, \bar{u}([0, T]))=\int_{0}^{T} l(\bar{x}(\tau), \bar{u}(\tau)) d \tau+m(\bar{x}(T)) .
$$

The finite horizon cost $J_{T}(\cdot)$ is composed of the stage cost $l: \mathbb{R}^{n} \times \mathbb{R}^{m} \rightarrow \mathbb{R}_{\geq 0}$ and the terminal cost $m: \mathbb{R}^{n} \rightarrow \mathbb{R}_{\geq 0}$, which is defined over the auxiliary terminal set $\mathcal{X}_{\text {aux }} \subseteq \mathbb{R}^{n}$. We denote by $k_{\text {aux }}: \mathcal{X}_{\text {aux }} \rightarrow \mathcal{U}$ an auxiliary control law defined over the terminal set.

In a sample-data receding horizon strategy, the control input is computed at discrete time samples

$$
\mathcal{T}:=\left\{t_{0}=0, t_{1}, \ldots\right\},
$$

where $t_{i}>t_{j}$ for $i>j$, and the MPC control law is defined as

$$
u(t)=k_{M P C}(x(t)):=\bar{u}^{*}(t-\lfloor t\rfloor ; x(\lfloor t\rfloor)),
$$

where $\lfloor t\rfloor$ is the maximum sampling instant $t_{i} \in \mathcal{T}$ smaller than or equal to $t$, i.e., $\lfloor t\rfloor=\max _{i \in \mathbb{N}_{\geq 0}}\left\{t_{i} \in \mathcal{T}: t_{i} \leq t\right\}$. Since the system is not time varying, the open-loop state and input trajectories $\bar{x}(\cdot)$ and $\bar{u}(\cdot)$, respectively, are considered, without loss of generality, in the interval $[0, T]$ and $t_{0}$ is chosen to be the time zero.

This work addresses the problem of designing an MPC controller to minimize an economic cost while guaranteeing asymptotic boundedness of the closed-loop trajectories around a given steady-state. Toward this goal, the stage cost is defined as

$$
l(x, u):=l_{s}(x, u)+l_{e}(x, u) .
$$

where the stabilizing stage cost $l_{s}: \mathbb{R}^{n} \times \mathbb{R}^{m} \rightarrow \mathbb{R}_{\geq 0}$ determines the attractiveness of the neighborhood of the given steady-state, as will be made clear later, and the economic stage cost $l_{e}: \mathbb{R}^{n} \times \mathbb{R}^{m} \rightarrow \mathbb{R}$ is an arbitrary economic function that we wish to minimize. In the following, we assume, without loss of generality, that the desired steadystate pair is $(0,0)$, which is always satisfied performing an appropriate change of state and input coordinates.

\section{MAIN RESULT}

Before stating the main result, we introduce the following assumptions that are common in the Tracking MPC literature.

Assumption 1: The function $f(\cdot)$, introduced in (1), is locally Lipschitz continuous in $x$ and piecewise continuous in $u$ in the region of interest. Moreover, without loss of generality, it satisfies $f(0,0)=0$.

Assumption 2 (Initial feasibility): The optimization problem $\mathcal{P}\left(x_{0}\right)$ admits a feasible solution.

Assumption 3 (Stabilizing stage cost):

(i) The state constraint set $\mathcal{X}$ and the terminal set $\mathcal{X}_{\text {aux }} \subseteq \mathcal{X}$ are closed, connected, and contain the origin. Moreover, the input constraint set $\mathcal{U}$ is compact with $0 \in \mathcal{U}$.

(ii) The stabilizing stage cost satisfies $l_{s}(0,0)=0$ and there is a class- $\mathcal{K}^{1}$ function $\alpha: \mathbb{R}_{\geq 0} \rightarrow \mathbb{R}_{\geq 0}$ such that $l_{s}(x, u) \geq \alpha(\|x\|)$ for all $(x, u) \in \mathbb{R}^{n} \times \mathbb{R}^{m}$.

(iii) The function $m(\cdot)$ is positive semi-definite and continuously differentiable away from the origin.

(iv) There exists a feasible auxiliary control law $k_{\text {aux }}: \mathcal{X}_{\text {aux }} \rightarrow \mathcal{U}$, defined over the terminal set $\mathcal{X}_{\text {aux }} \subseteq \mathbb{R}^{n}$, such that, for the closed-loop system (1) with $u(t)=k_{\text {aux }}(x)$, the state and input vectors satisfy $x(t) \in \mathcal{X}_{\text {aux }} \subseteq \mathcal{X}$ and $u(t) \in \mathcal{U}$, respectively, and the following condition holds:

$$
\dot{m}(x)=m_{x}(x) f\left(x, k_{\text {aux }}(x)\right) \leq-l_{s}\left(x, k_{\text {aux }}(x)\right)
$$

for all $x \neq 0$ and initial conditions $x_{0} \in \mathcal{X}_{a u x}$.

The notation $m_{x}(\cdot)$ denotes the partial derivatives of $m(\cdot)$ with respect of the state variable $x$.

Remark 1: (Design methods) It is worth noticing that setting $l_{e}(\cdot)=0$, Assumption 3 coincides with well known sufficient conditions for convergence to the origin of the MPC strategy, see, e.g., [9], [14]. This allows to use standard design techniques to compute a suitable terminal set and terminal cost.

Since the goal is to steer the state to bounded region around the origin, the following assumption is used to avoid the economic stage cost $l_{e}(\cdot)$ to constantly dominate the stabilizing stage cost $l_{s}(\cdot)$.

Assumption 4 (Bound on the economic stage cost): The norm of the economic stage cost function $l_{e}(\cdot)$, evaluated along the closed-loop state and input trajectories, is uniformly bounded by a strictly positive constant value, i.e., $\left\|l_{e}(x(t), u(t))\right\| \leq B, \forall t \geq 0$ with $B>0$.

Remark 2 (Design of the economic stage cost): Consider a given generic economic stage cost $\bar{l}_{e}: \mathbb{R}^{n} \times \mathbb{R}^{m} \rightarrow \mathcal{E}$ that we wish to minimize. For the case of bounded set $\mathcal{E}$, Assumption 4 , with $l_{e}(x, u)=\bar{l}_{e}(x, u)$, is trivially satisfied. In the general case, i.e, $\mathcal{E}=\mathbb{R}$, where Assumption 4 can be difficult to be a priori verified, a possible approach consists in applying a smooth saturation-like function and design $l_{e}(\cdot)=k \operatorname{atan}\left(\frac{1}{k} \bar{l}_{e}(\cdot)\right)$, where the constant

\footnotetext{
${ }^{1}$ A function $\alpha: \mathbb{R}_{\geq 0} \rightarrow \mathbb{R}_{\geq 0}$ is said to be belong to class $\mathcal{K}$, or to be a class- $\mathcal{K}$ function, if it is zero at zero, strictly increasing and radially unbounded, i.e., $\alpha(x) \rightarrow \infty$ as $x \rightarrow \infty$.
} 
$k>0$ determines the size of the domain where the mapping behaves almost linearly. Such smooth saturation guarantees the stabilizing stage cost to eventually dominate the economic cost, as $\|x\| \rightarrow+\infty$, with the drawback of modifying the original economic function $\bar{l}_{e}(\cdot)$.

The main result of this work follows:

Theorem 1 (Ultimate boundedness): Consider system (1) in closed-loop with (5), where $l(\cdot)$ is decomposed as in (6), and suppose that Assumptions 1-4 hold. Then, for every $x_{0}$ that satisfy Assumption 2 the closed-loop state trajectory

- is uniformly bounded over time, i.e, $\|x(t)\| \leq c$, with $c \in[0,+\infty)$, for all $t \geq 0$, and

- converges to an ultimate bound with size proportional to the value of $B$ from Assumption 4, i.e., there exist a finite time $\bar{T} \geq 0$ and a constant $U>0$ such that

$$
\|x(t)\| \leq U, \quad \forall t \geq \bar{T}
$$

where, for every desired value of $U>0$ there exist a bound $B>0$ such that (8) holds.

\section{A. Proof of Theorem 1}

Consider the standard value function:

Definition 2 (Value function): Consider a vector $\hat{x}$ and let the optimization problem $\mathcal{P}(\hat{x})$ admit a feasible solution. Using the minimizer $\bar{u}^{*}(\cdot ; \hat{x})$, and the associated state trajectory $\bar{x}^{*}(\cdot ; \hat{x})$, the value function is defined as

$$
V(\hat{x}):=\int_{0}^{T} l\left(\bar{x}^{*}, \bar{u}^{*}\right) d \tau+m\left(\bar{x}^{*}(T)\right) .
$$

The proof is structured as follows: first, a bound on the decrease of the value function is derived in Lemma 1. Then, ultimate boundedness of a generic sampling of the closedloop trajectory is shown in Lemma 3. Finally, we show by contradiction that the same ultimate boundedness applies to the whole closed-loop trajectory, which concludes the proof.

In the following, for a generic function $V(\cdot)$ and a scalar $\gamma$ the term $\mathcal{L}(V, \gamma)$ denotes the $\gamma$-sublevel set of $V(\cdot)$, i.e., $\mathcal{L}(V, \gamma):=\{x: V(x) \leq \gamma\}$. Moreover, we denote by $\mathcal{B}(r)$ a ball set of radius $r$ defined as $\mathcal{B}(r):=\{x:\|x\| \leq r\}$.

Lemma 1: Suppose that Assumptions 3-4 hold. Then, along the closed-loop (1) with (5) and for any $\hat{t} \geq 0$ and $\delta \geq 0$, the following cost inequality holds :

$$
V(x(\hat{t}+\delta)) \leq V(x(\hat{t}))-\int_{\hat{t}}^{\hat{t}+\delta} \alpha(\|x(\tau)\|) d \tau+2 \delta B
$$

where $V(\cdot)$ is the value function of Definition 2 .

Proof: This proof follows from standard arguments and is omitted due to space constraints. See, e.g., the work [3], where as only difference, the effect of the bounded economic stage cost results in the additive term $2 \delta B$ of (10).

Note that the Lemma 1 applies for any of $\delta>0$, which is independent from the choice of the sampling instants $\mathcal{T}$ in (4). Thus, for the generic sampling of the closed-loop trajectory defined as

$$
\mathcal{S}:=\left\{s_{i}=x\left(\xi_{i}\right) \text { with } \xi_{i}=\delta i, i \in \mathbb{N}_{\geq 0}\right\}
$$

we can write

$$
V\left(s_{i+1}\right) \leq V\left(s_{i}\right)-\int_{\xi_{i}}^{\xi_{i}+\delta} \alpha(\|x(\tau)\|) d \tau+2 \delta B .
$$

The following result will be needed in the proof of Lemma 3 on the ultimate boundedness of the generic sampling $\mathcal{S}$.

Lemma 2: (Ultimate bound) Let Assumption 1 hold and let $\phi\left(t ; t_{0}, x_{0}\right)$ denote a continuous solution of (1) starting at $x_{0}=x\left(t_{0}\right)$. Then, there exists a $\bar{\Delta}>0$ such that, for any class- $\mathcal{K}$ function $\alpha(\cdot)$ and scalars $b$ and $\Delta \leq \bar{\Delta}$, the set

$$
\mathcal{G}_{\Delta}=\left\{x_{0}: \min _{\tau \in\left[t_{0}, t_{0}+\Delta\right]} \alpha\left(\left\|\phi\left(\tau ; t_{0}, x_{0}\right)\right\|\right) \leq b\right\},
$$

which coincides with set of initial states of closed-loop state trajectories that are driven through $\mathcal{L}(\alpha(\|\cdot\|), b)$ in the interval of time $[t, t+\Delta]$, can be made arbitrarily small by reducing the value of $\Delta>0$ and $b>0$.

Proof: Start by noticing that $\mathcal{G}_{\Delta}$ consists in the set of initial conditions $x_{0}=x\left(t_{0}\right)$ that can be steered through the set

$$
\mathcal{L}(\alpha(\|\cdot\|), b)=\{x: \alpha(\|x\|) \leq b\}
$$

within a finite time $\Delta$. Then, the distance of a generic point $x_{0} \in \mathcal{G}_{\Delta}$ to the origin, i.e., $\left\|x_{0}\right\|$, with $x_{0} \in \mathcal{G}_{\Delta}$, can be bounded by the sum of the distance from $x_{0}$ to the set (14) plus the radius of the smallest ball containing the set (14), i.e., $r_{b}:=\alpha^{-1}(b)$, that always exists from $\alpha(\cdot)$ being a class$\mathcal{K}$ function and, thus, (14) being compact.

By the locally Lipschitz property from Assumption 1 there exists a $\bar{\Delta}>0$ such that the trajectory $\phi\left(\left[t_{0}, t_{0}+\bar{\Delta}\right] ; t_{0}, x_{0}\right)$ exists and has unique solution (Theorem 3.1 of [20]). Consider $\Delta<\bar{\Delta}$ and let $L_{\Delta}$ be the length of the trajectory $\phi\left(\left[t_{0}, t_{0}+\Delta\right] ; t_{0}, x_{0}\right)$, i.e., the piece of trajectory $\phi\left(\tau ; t_{0}, x_{0}\right)$, evaluated with $\tau \in\left[t_{0}, t_{0}+\Delta\right]$. Then, the distance from $x_{0} \in \mathcal{G}_{\Delta}$ to the set (14) is clearly less than the length $L_{\Delta}$.

Therefore, for all $x_{0} \in \mathcal{G}_{\Delta}$ we have $\left\|x_{0}\right\| \leq r_{b}+L_{\Delta}$ and, thus, $\mathcal{G}_{\Delta} \subseteq \mathcal{B}\left(r_{b}+L_{\Delta}\right)$. Moreover, note that the value of $r_{b}$ can be made arbitrarily small by decreasing the value of $b$ and, by continuity of the solution $\phi(\cdot), L_{\Delta}$ can be made arbitrarily small by decreasing the value of $\Delta$, which concludes the proof.

Lemma 3: (Ultimate boundedness of a sampling) Consider system (1) in closed-loop with (5) and suppose that Assumptions 1-4 hold. Moreover, consider a sampling $\mathcal{S}$ of the closed-loop state trajectory defined in (11) with $\delta<\Delta$. Then, there exists a $\bar{\Delta}>0$ such that for finite constants $\Delta \in(0, \bar{\Delta}]$ and $B>0$

- $\left\|s_{i}\right\| \leq c$ with $c \in[0,+\infty)$, for all $s_{i} \in \mathcal{S}$ and

- there exist a finite time $\bar{T} \geq 0$ and a constant $U>0$ such that

$$
\left\|s_{i}\right\| \leq U, \quad \forall \xi_{i} \geq \bar{T}
$$

where, for every value of $U>0$ there exist a bound $B>0$, from Assumption 4, such that (15) holds. 
Proof: From (12) and for any constant $\theta \in(0,1)$ we can write

$$
\begin{aligned}
& V\left(s_{i+1}\right)-V\left(s_{i}\right) \\
& \leq-(1-\theta) \int_{\xi_{i}}^{\xi_{i}+\delta} \alpha(\|x(\tau)\|) d \tau, \forall s_{i} \in \mathcal{D}_{i} \\
& \leq-(1-\theta) 2 \delta B / \theta, \forall s_{i} \in \mathcal{D}_{i}
\end{aligned}
$$

with

$$
\mathcal{D}_{i}:=\left\{s_{i}=x\left(\xi_{i}\right):-\theta \int_{\xi_{i}}^{\xi_{i}+\delta} \alpha(\|x(\tau)\|) d \tau+2 \delta B \leq 0\right\}
$$

and where the last inequality follows from $s_{i} \in \mathcal{D}_{i}$ and, thus, $2 \delta B / \theta \leq \int_{\xi_{i}}^{\xi_{i}+\delta} \alpha(\|x(\tau)\|) d \tau$.

For the generic set $A$, let $A^{c}$ denote the complement set of $A$, thus $\mathcal{D}_{i}^{c}=\left\{x\left(\xi_{i}\right): \theta \int_{\xi_{i}}^{\xi_{i}+\delta} \alpha(\|x(\tau)\|) d \tau<2 \delta B\right\}$. Note that $\mathcal{D}_{i}^{c} \subseteq\left\{x\left(\xi_{i}\right): \theta \delta \min _{\tau \in\left[\xi_{i}, \xi_{i}+\delta\right]} \alpha(\|x(\tau)\|) \leq 2 \delta B\right\} \subseteq$ $\mathcal{U}_{B \Delta}$ with

$$
\mathcal{U}_{B \Delta}:=\left\{x_{0}: \min _{\tau \in\left[t_{0}, t_{0}+\Delta\right]} \alpha(\|x(\tau)\|) \leq 2 B / \theta\right\},
$$

where the first inclusion follows from $\alpha(\cdot)$ being positive and, thus, $\int_{\xi_{i}}^{\xi_{i}+\delta} \alpha(\|x(\tau)\|) d \geq \delta \min _{\tau \in[\delta i, \delta(i+1)]} \alpha(\|x(\tau)\|)$, and the second inclusion is obtained from definition (17) and the inequality $\min _{\tau \in\left[\xi_{i}, \xi_{i}+\delta\right]} \alpha(\|x(\tau)\|) \geq \min _{\tau \in\left[\xi_{i}, \xi_{i}+\Delta\right]} \alpha(\|x(\tau)\|)$ resulting from the increase of the feasible set for $\tau$.

Note that, choosing $\Delta<\bar{\Delta}$ from Lemma 2 with $b=2 B / \theta$, the set $\mathcal{U}_{B \Delta}$ is bounded.

From the boundedness of $\mathcal{U}_{B \Delta}$ there always exists the bounded level set $\mathcal{L}\left(V, \gamma^{\prime}\right)$, with $\gamma^{\prime}=\min _{\bar{\gamma}: \mathcal{U}_{B} \subseteq \mathcal{L}(V, \bar{\gamma})}$, such that $\mathcal{U}_{B \Delta} \subseteq \mathcal{L}\left(V, \gamma^{\prime}\right)$. Thus, from (16), we obtain

$$
V\left(s_{i+1}\right)-V\left(s_{i}\right) \leq-(1-\theta) 2 \delta B / \theta, \forall s_{i} \notin \mathcal{L}\left(V, \gamma^{\prime}\right)
$$

which, by recursion, implies that $s_{i}$ enters the set $\mathcal{L}\left(V, \gamma^{\prime}\right)$ in, at most, the finite number of steps $k \geq \theta \frac{V\left(x_{0}\right)-\gamma^{\prime}}{(1-\theta) 2 \delta B}$.

Note that (18) does not describe the behavior of the value function $V(\cdot)$ once $s_{i} \in \mathcal{L}\left(V, \gamma^{\prime}\right)$, although (10) can be used to obtain a bound on the increase of $V(\cdot)$ as

$$
V\left(s_{i+1}\right)-V\left(s_{i}\right) \leq 2 \delta B \leq 2 \Delta B, \forall s_{i} \in \mathcal{L}\left(V, \gamma^{\prime}\right) .
$$

Combining (18) and (19), we obtain the bound $V\left(s_{i}\right) \leq \gamma:=\gamma^{\prime}+2 \Delta B, \forall k \geq \theta \frac{V\left(s_{0}\right)-\gamma}{(1-\theta) 2 \delta B}$ and, consequently, $s_{i} \in \mathcal{L}(V, \gamma), \forall \xi_{i}=\delta k \geq \theta \frac{V\left(s_{0}\right)-\gamma}{(1-\theta) 2 B}=\bar{T}$. Now let $\mathcal{B}(U)$ be the smallest ball containing $\mathcal{L}(V, \gamma)$, that always exists from $\mathcal{L}(V, \gamma)$ being compact, then we have $\left\|s_{i}\right\| \leq U, \forall \xi_{i} \geq \bar{T}$.

Note that, using Lemma 2 and the definition of $U$, the value of $U$ can be made arbitrarily small by reducing $B>0$ and $\Delta>0$. Moreover, note that the samples $\mathcal{S}$ are always bounded. In fact, also during the first phase, where the sampling is converging to the ultimate bound, the Lyapunov decrease (18) implies that $s_{i} \in \mathcal{L}\left(V, V\left(s_{0}\right)\right)$. This concludes the proof.
It is important to notice that, in Lemma 3, the variable $\bar{T}$ and $U$ are independent from the chosen sampling, i.e, it holds for any value of $\delta \leq \Delta$. This fact is used to proof, by contradiction, that the whole closed-loop trajectory satisfies the same property (first the statement of Theorem 1 is negated, then we show that this leads to a contradiction).

Negating Theorem 1 results in: there exists an $x_{0}$, satisfying Assumption 2, such that for the closed-loop trajectory

- there exist a $t_{1}$ such that $\left\|x\left(t_{1}\right)\right\|>c$ for any $c \in[0,+\infty)$, or

- for all time $\bar{T} \geq 0$ and a constants $U>0$ there exists a $t_{2} \geq \bar{T}$ for which $\left\|x\left(t_{2}\right)\right\|>U$.

However,

- by choosing $\delta$ small enough such that $\delta i=t_{1}$, we have by Lemma 3 , that $\left\|x\left(t_{1}\right)\right\|=\left\|s_{i}\right\| \leq c$ with $c \in[0,+\infty)$, which contradicts the first point.

- Moreover, by choosing $U \geq 0$ and $\bar{T} \geq 0$ from Lemma 3 , and $\delta$ small enough such that $\bar{T} \leq \xi_{i}=\delta i=t_{2}$ for some $i$, implies that $\|x(\bar{t})\| \leq U$, which contradicts the second point.

This concludes the proof.

\section{SIMULATION RESULTS}

Consider a follower vehicle described by the unicycle model $\dot{p}=\left(\begin{array}{l}v \cos (\theta) \\ v \sin (\theta)\end{array}\right)$ with $\dot{\theta}=\omega$, where $p \in \mathbb{R}^{2}$ and $\theta \in \mathbb{R}$ denote the position and the heading of the vehicle, and $v \in \mathbb{R}^{2}$ and $\omega \in \mathbb{R}$ denote its linear and angular velocity, respectively. The input $u:=(v, \omega)^{\prime} \in \mathcal{U}$, is considered to be constrained in the set $\mathcal{U}=\left\{(v, \omega)^{\prime}:-2 \leq v \leq 2,-\pi \leq \omega \leq \pi\right\}$. Moreover, consider a target vehicle described by a single integrator $\dot{p}_{t}(t)=u_{t}(t)$ with $\dot{u}_{t}(t)=0$ where $p_{t} \in \mathbb{R}^{2}$ and $u_{t} \in \mathbb{R}^{2}$ are the position and velocity of the target vehicle, respectively.

The position of the target vehicle is continuously observed by an omnidirectional camera centered at the position of the follower vehicle. Then, the perspective observation model (see, e.g., [2]) is defined as $y=\frac{p_{t}-p}{\left\|p_{t}-p\right\|}$, where $y \in \mathbb{R}^{2}$ is a bearing only observation $\left(\|y(t)\|=1, \forall p_{t} \neq p\right)$.

Assuming given a trajectory-tracking controller, a common approach to target-tracking consists in using the signals $u(\cdot)$ and $y(\cdot)$ to estimate the position and the direction of the target vehicle, and then to feed the estimate to the tracking controller that derives the input signal for the follower.

Although, for the case of nonlinear observation models, it can occur that the system is steered through unobservable trajectories, leading to an incorrect estimate of the target and, therefore, to the failure of the target-tracking strategy.

We address this problem by augmenting an MPC controller for trajectory-tracking with an economic stage cost used to penalizes unobservable trajectories. For the purposes of this paper, we only consider the state feedback case, with the known state of the target, and we refer to [6] for a the case with general vehicle models, output feedback, and state estimation via Extended Kalman Filter.

In the following simulations, we consider the set of sampling instants $\mathcal{T}=\left\{t_{i}=0.1 i, i \in \mathbb{N}_{\geq 0}\right\}$ and the 


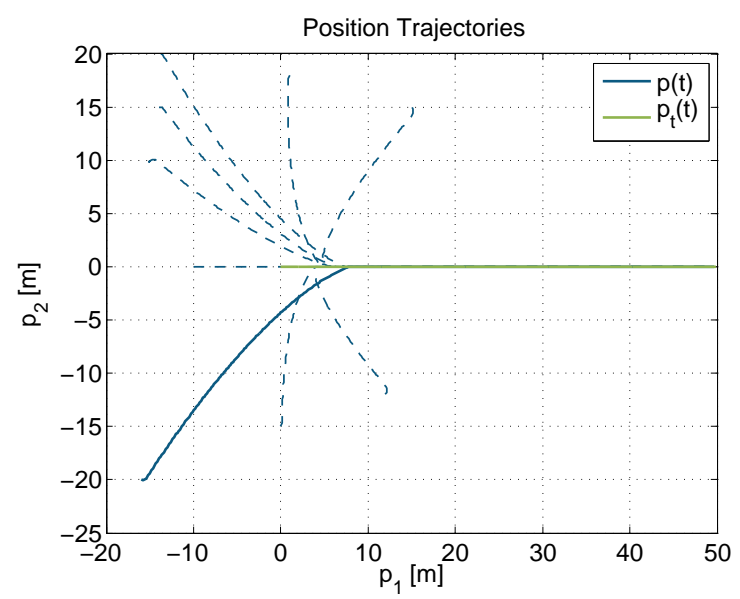

Fig. 1. The solid green line denotes the trajectory of the position of the target vehicle while the blue lines denote the closed-loop position trajectories of the follower vehicle starting at different initial conditions. The solid blue line highlights the trajectory associate with the initial condition $x_{0}=(-16,-20,-0.1)^{\prime}$.

horizon length $T=0.5 \mathrm{~s}$. Moreover, the MPC optimization problems are solved using the ACADO Toolkit [18].

\section{A. MPC for Trajectory-tracking}

Consider the state feedback trajectory-tracking controller from [4] where the trajectory to be followed is defined by a desired position, as function of time, its derivative, and a bound on such derivative. Since in this example we wish to track the position of the target vehicle, the target position, its derivative, and an a priori bound on the target velocity (which is considered to be $0.5 \mathrm{~m} / \mathrm{s}$ ) are used as input of the trajectory-tracking algorithm. Using the same notation in [4], the MPC controller parameters are chosen to be $\epsilon=(-0.2,0)^{\prime}, K=0.1 I_{2 \times 2}, O=0.1 I_{2 \times 2}$, and $Q=10 I_{2 \times 2}$, where $I_{2 \times 2}$ denotes an identity matrix of size $2 \times 2$, and the resulting terminal set is $\mathcal{E}_{f}=\left\{e^{\prime} e \leq 26.4^{2}\right\}$.

Fig. 1 displays the position of the system in closedloop with the resulting target-tracking controller for different initial conditions.

\section{B. The Economic Cost}

In this subsection, we identify a performance index that penalizes weakly observable trajectories using the properties of the observability matrix:

$$
\mathcal{O}(x, u)=\frac{\partial}{\partial\left(\begin{array}{l}
p_{t} \\
u_{t}
\end{array}\right)}\left(\begin{array}{c}
y(x) \\
\dot{y}(x, u) \\
\vdots
\end{array}\right)
$$

To provide an intuition of the meaning of (20), the observability matrix $\mathcal{O}(x, u)$, evaluated at the point $(x, u)$, describes the sensitivity of the output and its derivatives to variations of $\left(p_{t}^{\prime}, u_{t}^{\prime}\right)^{\prime}$ that wish to estimate. Thus, if the matrix is not full rank there exists a variation of $\left(p_{t}^{\prime}, u_{t}^{\prime}\right)^{\prime}$ that does not influence the output and its derivatives, and therefore the pair $\left(p_{t}^{\prime}, u_{t}^{\prime}\right)^{\prime}$ is locally not observable at $(x, u)$. Whereas for the case of linear systems it is enough to stop at the $(n-1)$-th derivative of the output, for a generic nonlinear (observable) system, a common approach consists in stopping when there exists at least a state and input pair that makes the matrix $\mathcal{O}$ full rank. In this example we stopped at the first derivative of the output.

Let $\sigma_{\min }(x, u)$ and $\sigma_{\max }(x, u)$ denote the minimum and the maximum singular value, respectively, of the observability matrix. The associated condition number $\kappa(x, u):=\sigma_{\max }(x, u) / \sigma_{\min }(x, u)$ provides a measure of the "quality" of the observability. In fact, broadly speaking, if the components of $x$ have the same "quality" of observability, we would have $\kappa(\mathcal{O}(x, u))=1$.

In view of these observations, the economic stage cost is designed as

$$
l_{e}(x, u)=10 k_{1} \operatorname{atan}\left(\frac{1}{k_{1}}\left(l_{e 1}(x, u)+l_{e 2}(x, u)\right)\right)
$$

with $l_{e 1}(x, u)=\left(10^{-2} \sigma_{\min }(x, u)+10^{-3}\right)^{-1}$ and $l_{e 2}(x, u)=10\left(\kappa(x, u)^{-1}-1\right)^{2}$ and $k_{1}=10^{4}$. Note that the cost $l_{e 1}(\cdot)$ strongly penalizes the case where the observability matrix loses rank, i.e., $\sigma_{\min }(x, u)=0$, and rewards the case of matrixes far from singularity, while the cost $l_{e 2}(\cdot)$ rewards observability matrices with good condition numbers, i.e., close to 1 . The economic cost $l_{e}(\cdot)$ is obtained combining the two costs and saturating them in order to meet the Assumption 4 on the bound of the economic cost, as suggested in Remark 2.

Fig. 3 (top), shows the economic stage cost evaluated along the closed-loop trajectories associated with the controller proposed in Section IV-A. Note that, the closed-loop trajectory are driven through poorly observable or not observable trajectories with the economic cost being saturated. This, in the case of output feedback, would cause a bad quality of the estimate and, thus, the failure of the output feedback strategy, as shown in [6].

\section{The Proposed Scheme}

Since the MPC controller in Subsection IV-A satisfies Assumption 1-3 and the economic stage cost computed in Subsection IV-B satisfies Assumption 4, the proposed scheme can be applied.

Fig. 2 displays the position trajectories of the system in closed-loop with the target-tracking controller augmented with the economic performance index. In this case, we obtain the desired behavior where the follower, first approaches the target, and then starts performing circular motions around it in order to keep a good estimate of its position and velocity. In fact, as effect of these maneuvers, Fig. 3 shows that, after an initial transient behavior, the economic stage cost never saturates, which implies the observability of the pair $\left(p_{t}^{\prime}, u_{t}^{\prime}\right)^{\prime}$.

\section{CONCLUSION AND FUTURE WORKS}

A sample-data state feedback MPC scheme with economic stage function is presented together with a set of sufficient conditions for convergence of the norm of the closed-loop state vector to an ultimate bound. The computation of a suitable terminal set and terminal cost can be performed using the standard design methods adopted in Tracking MPC and 


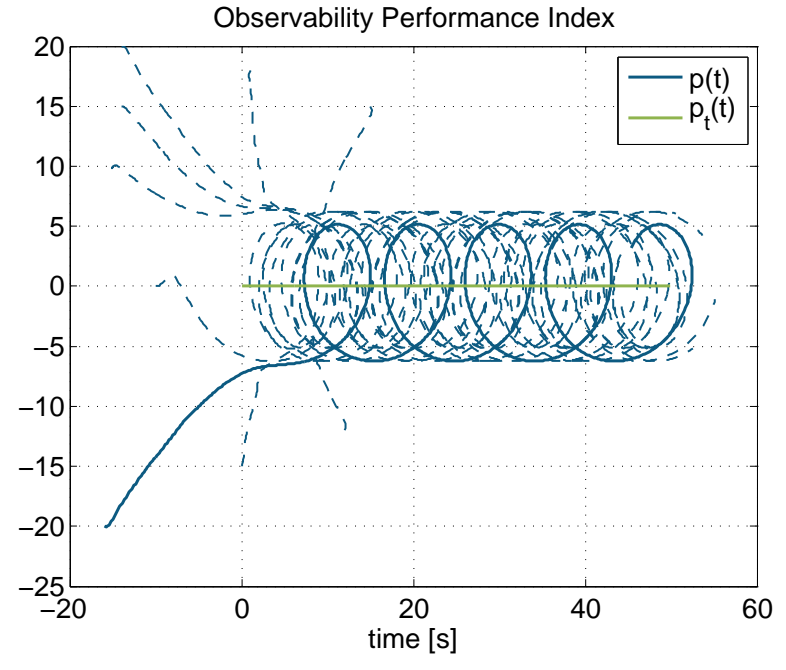

Fig. 2. The solid green line denotes the trajectory of the position of the target vehicle while the blue lines denote the closed-loop position trajectories of the follower vehicle starting at different initial conditions. The solid blue line highlights the trajectory associate with the initial condition $x_{0}=(-16,-20,-0.1)^{\prime}$.

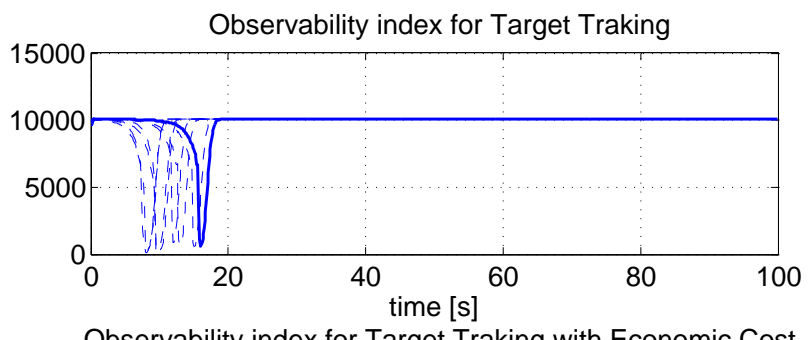

Observability index for Target Traking with Economic Cost

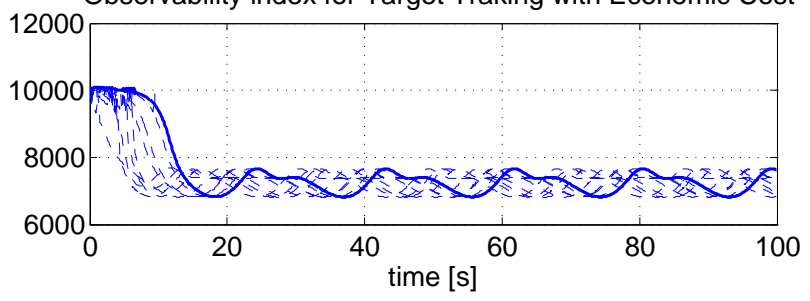

Fig. 3. The economic cost computed along the closed-loop input and state trajectories associated with the target-tracking MPC controller and targettracking MPC controller with additional economic cost are displayed on the top and the bottom figure, respectively, from different initial conditions. With solid lines, we denote the trajectories associated with the initial condition $x_{0}=(-16,-20,-0.1)^{\prime}$.

a suitable economic cost can be enforced, if needed, using saturation-like functions. The flexibility of minimizing an economic cost while still bounding the asymptotic behavior of the closed-loop trajectories around a desired steady-state gives space to many practical applications. As an example, the proposed scheme is applied to address a target estimation and tracking control problem.

\section{REFERENCES}

[1] A. Aguiar and M. Bayat. Observability analysis of the simultaneous underwater vehicle localization and mapping based on ranging measurement. In Proc. of the 10th Portuguese Conf. on Automatic Control, Controlo, number July, 2012.
[2] A. Aguiar and J. Hespanha. Minimum-Energy State Estimation for Systems With Perspective Outputs. IEEE Trans. on Automatic Control, 51(2):226-241, Feb. 2006.

[3] A. Alessandretti, A. Aguiar, and C. Jones. A Model Predictive Control Scheme with Additional Performance Index for Transient Behavior. In Proc. of the 52nd Conf. on Decision and Control, pages 5090-5095, Florence, Italy, 2013.

[4] A. Alessandretti, A. Aguiar, and C. Jones. Trajectory-tracking and path-following controllers for constrained underactuated vehicles using Model Predictive Control. In Proc. of the 2013 European Control Conference, pages 1371-1376, 2013.

[5] A. Alessandretti, A. P. Aguiar, and C. Jones. An Economic Model Predictive Control scheme with terminal penalty for continuous-time systems. In Proc. of the 53th Conf. on Decision and Control, 2014.

[6] A. Alessandretti, A. P. Aguiar, and C. N. Jones. Optimization based control for target estimation and tracking via highly observable trajectories. Proc. of 11th Portuguese Conf. on Automatic Control, Controlo, 2014.

[7] R. Amrit, J. B. Rawlings, and D. Angeli. Economic optimization using model predictive control with a terminal cost. Annual Reviews in Control, 35(2):178-186, Dec. 2011.

[8] D. Angeli, R. Amrit, and J. B. Rawlings. On Average Performance and Stability of Economic Model Predictive Control. IEEE Trans. on Automatic Control, 57(7):1615-1626, July 2012.

[9] H. Chen and F. Allgöwer. A Quasi-Infinite Horizon Nonlinear Model Predictive Control Scheme with Guaranteed Stability. Automatica, 34(10):1205-1217, Oct. 1998.

[10] J. Cochran and M. Krstic. Nonholonomic Source Seeking With Tuning of Angular Velocity. IEEE Trans. on Automatic Control, 54(4):717731, 2009.

[11] M. Diehl, R. Amrit, and J. B. Rawlings. A Lyapunov Function for Economic Optimizing Model Predictive Control. IEEE Trans. on Automatic Control, 56(3):703-707, Mar. 2011

[12] L. Fagiano and A. R. Teel. Generalized terminal state constraint for model predictive control. Automatica, 49(9):2622-2631, Sept. 2013.

[13] A. Ferramosca, J. Rawlings, D. Limon, and E. Camacho. Economic MPC for a changing economic criterion. In Proc. of the 49th Conf. on Decision and Control, pages 6131-6136. IEEE, Dec. 2010.

[14] F. A. Fontes. A general framework to design stabilizing nonlinear model predictive controllers. Systems \& Control Letters, 42(2):127143, Feb. 2001.

[15] L. Grüne. Economic receding horizon control without terminal constraints. Automatica, 49(3):725-734, Mar. 2013.

[16] L. Grune and J. Pannek. Nonlinear model predictive control: theory and algorithms. 2011.

[17] M. Heidarinejad, J. Liu, and P. D. Christofides. Economic model predictive control of nonlinear process systems using Lyapunov techniques. AIChE Journal, 58(3):855-870, Mar. 2012.

[18] B. Houska, H. J. Ferreau, and M. Diehl. ACADO toolkit-An opensource framework for automatic control and dynamic optimization. Optimal Control Applications and Methods, 32(3):298-312, 2011.

[19] A. Jadbabaie, J. Yu, and J. Hauser. Unconstrained receding-horizon control of nonlinear systems. IEEE Trans. on Automatic Control, 46(5):776-783, May 2001.

[20] H. K. Khalil. Nonlinear Systems. Prentice Hall PTR, 2002.

[21] J. P. Maree and L. Imsland. Performance and Stability for Combined Economic and Regulatory Control in MPC. In Proc. of the 19th World Congress The International Federation of Automatic Control, 2014.

[22] D. Mayne, J. Rawlings, C. Rao, and P. Scokaert. Constrained model predictive control: Stability and optimality. Automatica, 36(6):789814 , June 2000

[23] M. A. Müller, D. Angeli, and F. Allgöwer. Economic model predictive control with self-tuning terminal cost. European Journal of Control, 19(5):408-416, Sept. 2013.

[24] O. Namaki-Shoushtari, A. Pedro Aguiar, and A. Khaki-Sedigh. Target tracking of autonomous robotic vehicles using range-only measurements: a switched logic-based control strategy. International Journal of Robust and Nonlinear Control, 22(17):1983-1998, 2012.

[25] S. A. P. Quintero, F. Papi, D. J. Klein, L. Chisci, and J. P. Hespanha. Optimal UAV coordination for target tracking using dynamic programming. In Proc. of the 49th Conf. on Decision and Control, pages 4541-4546, Dec. 2010.

[26] J. B. Rawlings and D. Q. Mayne. Model Predictive Control Theory and Design. Nob Hill Pub., 2009. 